\title{
Missense variant in CCDC22 causes X-linked recessive intellectual disability with features of Ritscher-Schinzel/3C syndrome
}

\section{This paper has been corrected since online publication and a corrigendum also appears in this issue.}

\author{
Mateusz Kolanczyk ${ }^{\star, 1,2}$, Peter Krawitz ${ }^{3}$, Jochen Hecht ${ }^{3}$, Anna Hupalowska ${ }^{4}$, Marta Miaczynska ${ }^{4}$, \\ Katrin Marschner ${ }^{1}$, Claire Schlack ${ }^{1}$, Denise Emmerich ${ }^{1,2}$, Karolina Kobus ${ }^{1}$, Uwe Kornak ${ }^{1}$, Peter N Robinson ${ }^{1}$, \\ Barbara Plecko ${ }^{5}$, Gernot Grangl ${ }^{6}$, Sabine Uhrig ${ }^{7}$, Stefan Mundlos ${ }^{1,2,3}$ and Denise Horn ${ }^{\star, 1}$
}

Ritscher-Schinzel syndrome (RSS)/3C (cranio-cerebro-cardiac) syndrome (OMIM\#220210) is a rare and clinically heterogeneous developmental disorder characterized by intellectual disability, cerebellar brain malformations, congenital heart defects, and craniofacial abnormalities. A recent study of a Canadian cohort identified homozygous sequence variants in the KIAAO196 gene, which encodes the WASH complex subunit strumpellin, as a cause for a form of RSS/3C syndrome. We have searched for genetic causes of a phenotype similar to RSS/3C syndrome in an Austrian family with two affected sons. To search for disease-causing variants, whole-exome sequencing (WES) was performed on samples from two affected male children and their parents. Before WES, CGH array comparative genomic hybridization was applied. Validation of WES and segregation studies was done using routine Sanger sequencing. Exome sequencing detected a missense variant (c.1670A $>$ G; p.(Tyr557Cys)) in exon 15 of the CCDC22 gene, which maps to chromosome Xp11.23. Western blots of immortalized lymphoblastoid cell lines (LCLs) from the affected individual showed decreased expression of CCDC22 and an increased expression of WASH1 but a normal expression of strumpellin and FAM21 in the patients cells. We identified a variant in CCDC22 gene as the cause of an X-linked phenotype similar to RSS/3C syndrome in the family described here. A hypomorphic variant in CCDC22 was previously reported in association with a familial case of syndromic X-linked intellectual disability, which shows phenotypic overlap with RSS/3C syndrome. Thus, different inactivating variants affecting CCDC22 are associated with a phenotype similar to RSS/3C syndrome.

European Journal of Human Genetics (2015) 23, 633-638; doi:10.1038/ejhg.2014.109; published online 11 June 2014

\section{INTRODUCTION}

Ritscher-Schinzel syndrome/3C (cranio-cerebro-cardiac) (RSS/3C) syndrome is a rare and presumably autosomal recessive intellectual disability (ID) syndrome with a specific pattern of malformations of the brain and heart as well as minor anomalies of the face and the hands and feet. Approximately $80 \%$ of RSS/3C syndrome patients show cardiac defects, which can include septal defects, tetralogy of Fallot, double outlet right ventricle, hypoplastic left heart, aortic stenosis, pulmonic stenosis, and other valvular anomalies. Many patients show Dandy-Walker malformation, cerebellar vermis hypoplasia, posterior fossa cysts, and ventricular dilatation. ${ }^{1}$ The facial dysmorphism of RSS/3C syndrome is characterized by a prominent forehead, prominent occiput, downslanting palpebral fissures, depressed nasal bridge, low-set ears, and micrognathia. The phenotypic manifestations of $\mathrm{RSS} / 3 \mathrm{C}$ syndrome can vary and cardiac and cerebellar manifestations are not always present. Therefore the pattern of craniofacial dysmorphic features is crucial in ascertaining the diagnosis. ${ }^{2,3}$ A recent study of a cohort of affected individuals of the First Nation community in northern Manitoba,
Canada, an isolate, identified homozygous sequence variants affecting the KIAA0196 gene, which encodes the WASH (Wiskott-Aldrich Syndrome Protein and SCAR Homolog) complex protein strumpellin as causal to a form of RSS/3C syndrome. ${ }^{2}$ Here we present a study of an Austrian family with two affected male children. To elucidate the molecular basis of their phenotype we applied NGS-based wholeexome sequencing (WES). In this report, we show that a missense variant in CCDC22 is associated with a form of X-linked ID with features of RSS/3C syndrome. Review of literature about CCDC22 (coiled-coil domain-containing protein 22) function and its molecular interactions points to a likely role of this protein both in the NF-kB signaling, and in the function of WASH complex, which is involved in actin polymerization and multiple endosomal transport processes.

\section{MATERIALS AND METHODS}

CGH array screening

In proband 1, array-CGH was performed with genomic DNA extracted from peripheral blood on an Agilent 60k Microarray (Agilent Technologies, Santa Clara, CA, USA). Slides were scanned using the Agilent microarray scanner G2505B and

\footnotetext{
${ }^{1}$ Institute for Medical Genetics and Human Genetics, Charité-Universitätsmedizin Berlin, Berlin, Germany; ${ }^{2}$ Max Planck Institute for Molecular Genetics, Berlin, Germany; ${ }^{3}$ Berlin-Brandenburg Center for Regenerative Therapies (BCRT), Charité-Universitätsmedizin Berlin, Berlin, Germany; ${ }^{4}$ Laboratory of Cell Biology, International Institute of Molecular and Cell Biology, Warsaw, Poland; ${ }^{5}$ Division of Child Neurology, University Children's Hospital Zurich, Zürich, Switzerland; ${ }^{6}$ Department of Pediatrics, Medical University Graz, Graz, Austria; ${ }^{7}$ Institute of Human Genetics, Medical University of Graz, Graz, Austria

*Correspondence: Dr M Kolanczyk or Professor D Horn, Institut für Medizinische Genetik und Humangenetik, Charité Universitätsmedizin, Campus Virchow, Augustenburger Platz 1, 13353 Berlin, Germany. Tel: +49 30450569 143; Fax: +49 30450569 915; E-mail: kolanshy@molgen.mpg.de (MK) or Tel: +49 30450569118 ; Fax: +49 30450569 914; E-mail: Denise.Horn@charite.de (DH)
}

Received 31 January 2014; revised 11 April 2014; accepted 16 April 2014; published online 11 June 2014 
images were analyzed using Agilent DNA Analytics software 4.0.76. The analysis with CGH analytics software was performed with the statistical algorithm ADM-2 with the sensitivity threshold set to 6.0. At least three consecutive oligos with a log ratio of 0.29 had to be aberrant to be called by the software.

\section{Whole-exome sequencing}

WES was done on DNA samples of the Austrian family (probands 1, 2 and their parents). Each DNA sample was captured using Agilent SureSelect Human All Exon Kit V2 and sequencing of 100-bp paired end reads was carried out on Illumina HiSeq 2000 (Illumina, Inc., San Diego, CA, USA). Sequence reads were aligned to the haploid human reference genome (hg 19) using Novoalign (Novocraft Technologies, Selangor, Malaysia). Single-nucleotide variants (SNVs) and short insertions and deletions (indels) were called using SAMtools. ${ }^{4}$ All detected variants were annotated with Annovar. ${ }^{5}$ Filtering of common variants present in the Exome Variant Server (EVS), dbSNP132 and the 1000 Genomes database, technical artifacts of sequencing, low-quality data $(\mathrm{Q}<60)$, and variants seen in more than three control exomes in our internal data set was performed using a set of Perl scripts. Finally, to predict pathogenic potential of missense variants we used PolyPhen 2 and Variant Taster. Only functionally relevant variants predicted to affect protein sequence, that is, coding non-synonymous SNVs, small indels, and splice site SNVs, were considered. Variants were visualized with use of integrative genomics viewer (IGV). ${ }^{6}$ Possible pathogenic variants were then validated with standard Sanger sequencing in all available family members and the CCDC22 variant was deposited in the Human and Clinical Genetics, Leiden University Medical Center - LOVD v.2.0 Build 35 database (URL: http://grenada.lumc.nl).

\section{Sanger sequencing}

Presence of the variant in $C C D C 22$ was confirmed with Sanger sequencing, which was done with dye terminator chemistry (ABI Prism BigDye, Life Technologies $\mathrm{GmbH}$, Darmstadt, Germany) and run on automated sequencer Applied Biosystems (Life Technologies GmbH) Prism 3700 DNA Analyzer as previously described. ${ }^{7}$ Sequences of the primers used for exon 15 amplification were:

CCDC22 ex15 F: $5^{\prime}$-ATGAAGCTAGTGGGGTGGTG-3'

CCDC22 ex15 R: 5'-CCACAGCCTCTGTCAGCATA-3'

\section{Western blots}

Whole cell lysates of patient and control lymphoblastoid cell lines (LCLs) were resolved by electrophoresis in SDS-polyacrylamide gels and transferred onto Optitran BA-S 85 membrane (GE Healthcare, Fairfield, CT, USA). For western blot analysis, membranes were probed with the antibodies:
anti-GAPDH AM4300 (Ambion, Life Technologies GmbH) 1:10000; anti-CCDC22 HPA000888 (Sigma-Aldrich GmbH, Seelze, Germany) 1:500; anti-Strumpellin sc-87442 (Santa Cruz Biotechnology, Inc., Dallas, TX, USA) 1:200; anti-FAM21 sc-137993 (Santa Cruz Biotechnology, Inc.) 1:100; and anti-WASH1 SAB4200372 (Sigma-Aldrich GmbH) 1:500.

\section{Transient transfection and immunofluorescence}

HeLa cells were grown on a 24-well plate on glass slides in Eagle's minimum essential medium (MEM) with $10 \%$ fetal bovine serum, $100 \mathrm{U} / \mathrm{ml}$ penicillin, $100 \mathrm{~g} / \mathrm{ml}$ streptomycin, and $2 \mathrm{~mm}$ glutamine (all reagents from Sigma-Aldrich $\mathrm{GmbH}$ ) at $37^{\circ} \mathrm{C}$ with $5 \% \mathrm{CO}_{2}$. Cells were transiently transfected with pCMV6-Flag-CCDC22 vectors (OriGene, Rockville, MD, USA) (WT, T17A Y557C), using $4 \mu \mathrm{l}$ polyethylenimine and $0.4 \mu \mathrm{g}$ DNA per well. After $24 \mathrm{~h}$, cells were serum-starved in MEM supplemented with $0.2 \%$ BSA for $4 \mathrm{~h}$ and stimulated with $500 \mathrm{ng} / \mathrm{ml}$ EGF-Rh (Invitrogen, Life Technologies $\mathrm{GmbH}$ ) for $20 \mathrm{~min}$. Following cargo uptake, cells were fixed with $3 \%$ paraformaldehyde in PBS for $12 \mathrm{~min}$ and permeabilized for $10 \mathrm{~min}$ with PBS containing $0.1 \% \mathrm{w} / \mathrm{v}$ saponin, $0.2 \% \mathrm{w} / \mathrm{v}$ gelatin, and $5 \mathrm{mg} / \mathrm{ml} \mathrm{BSA}$. Cells were further incubated with EEA1 (BD Transduction Laboratories, San Jose, CA, USA) and Flag (SigmaAldrich $\mathrm{GmbH}$ ) primary monoclonal antibodies. After washing with PBS, cells were incubated with Alexa-labeled secondary antibodies for $30 \mathrm{~min}$. Cells were washed and mounted with Mowiol (Sigma-Aldrich GmbH). Images were acquired with Leica TCS SP2 microscope (Leica Microsystems Inc., Buffalo Grove, IL, USA) equipped with AOBS using $\times 63 / 1.4$ NA oil immersion objective. The presented microscopy images were assembled using Adobe Photoshop CS5 (Adobe Systems Incorporated, San Jose, CA, USA)

\section{RESULTS}

The affected 8- and 15-year-old brothers are offspring of nonconsanguineous healthy parents of Austrian origin. They were born with normal measurements but they developed postnatal growth delay ( $-3.7 \mathrm{SD}$ in patient 1 and $-2.1 \mathrm{SD}$ in patient 2 ), and a relative large head circumference (occipitofrontal head circumference of $+1.3 \mathrm{SD}$ and $+0.8 \mathrm{SD}$, see Table 1). After birth, large anterior fontanelles were documented in both and cryptorchidism was noted in the older one. In addition, both had Dandy-Walker malformations and ventricular septal defects (VSD) for which corrective surgery was performed. Muscular hypotonia and psychomotor delay were noted in both from early infancy. Independent walking was achieved at ages of 21 and 22 months, respectively. At current age they are able to

Table 1 Summary of clinical findings in patients with CCDC22 variants and KIAA0196 variants

\begin{tabular}{|c|c|c|c|c|}
\hline & Patient 1 & Patient 2 & Voineagu et al ${ }^{9}$ & Elliott et $a l^{2}$ \\
\hline Variants & CCDC22 variant c. $1670 \mathrm{~A}>\mathrm{G}$ & CCDC22 variant c. $1670 A>G$ & $C C D C 22$ variant c. $219 \mathrm{~A}>\mathrm{G}$ & $\begin{array}{l}\text { Sequence variants of the } \\
\text { KIAAO196 gene }\end{array}$ \\
\hline Intellectual disability & + & + & + & + \\
\hline Postnatal growth retardation & $+(-3.7 \mathrm{SD})$ & $+(-2.1 \mathrm{SD})$ & n.d. & - \\
\hline Cerebellar malformations & Dandy-Walker malformation & Dandy-Walker malformation & $\begin{array}{l}\text { Dandy-Walker malformation, defects of } \\
\text { posterior fossa, cerebellar hypoplasia }\end{array}$ & $\begin{array}{l}\text { Structural posterior fossa } \\
\text { malformations }\end{array}$ \\
\hline Cardiac defects & VSD & VSD & ASD, VSD, dextrocardia & VSD, ASD, Tetralogy of Fallot \\
\hline \multicolumn{5}{|l|}{ Craniofacial } \\
\hline Head circumference & $\begin{array}{l}\text { Relative macrocephaly (OFC } \\
+1.3 \mathrm{SD})\end{array}$ & $\begin{array}{l}\text { Relative macrocephaly (OFC } \\
+0.8 \mathrm{SD})\end{array}$ & n.d. & Macrocephaly \\
\hline Hypertelorism & + & + & + & + \\
\hline Large anterior fontanelle & + & + & n.d. & + \\
\hline Limb anomalies & $\begin{array}{c}\text { Camptodactyly, reduced } \\
\text { distal creases, overriding toes }\end{array}$ & $\begin{array}{c}\text { Camptodactyly, reduced } \\
\text { distal creases, overriding toes }\end{array}$ & Absent distal creases, overriding toes & $\begin{array}{c}\text { Absent distal creases, syndactyly, } \\
\text { brachydactyly, camptodactyly }\end{array}$ \\
\hline Other & Cryptorchidism, glaucoma & - & Cryptorchidism, aplasia cutis, hypodontia & Coloboma \\
\hline
\end{tabular}

Abbreviations: ASD, atrial septal defect; OFC, occipitofrontal head circumference; VSD, ventricular septal defect; n.d., not documented. 

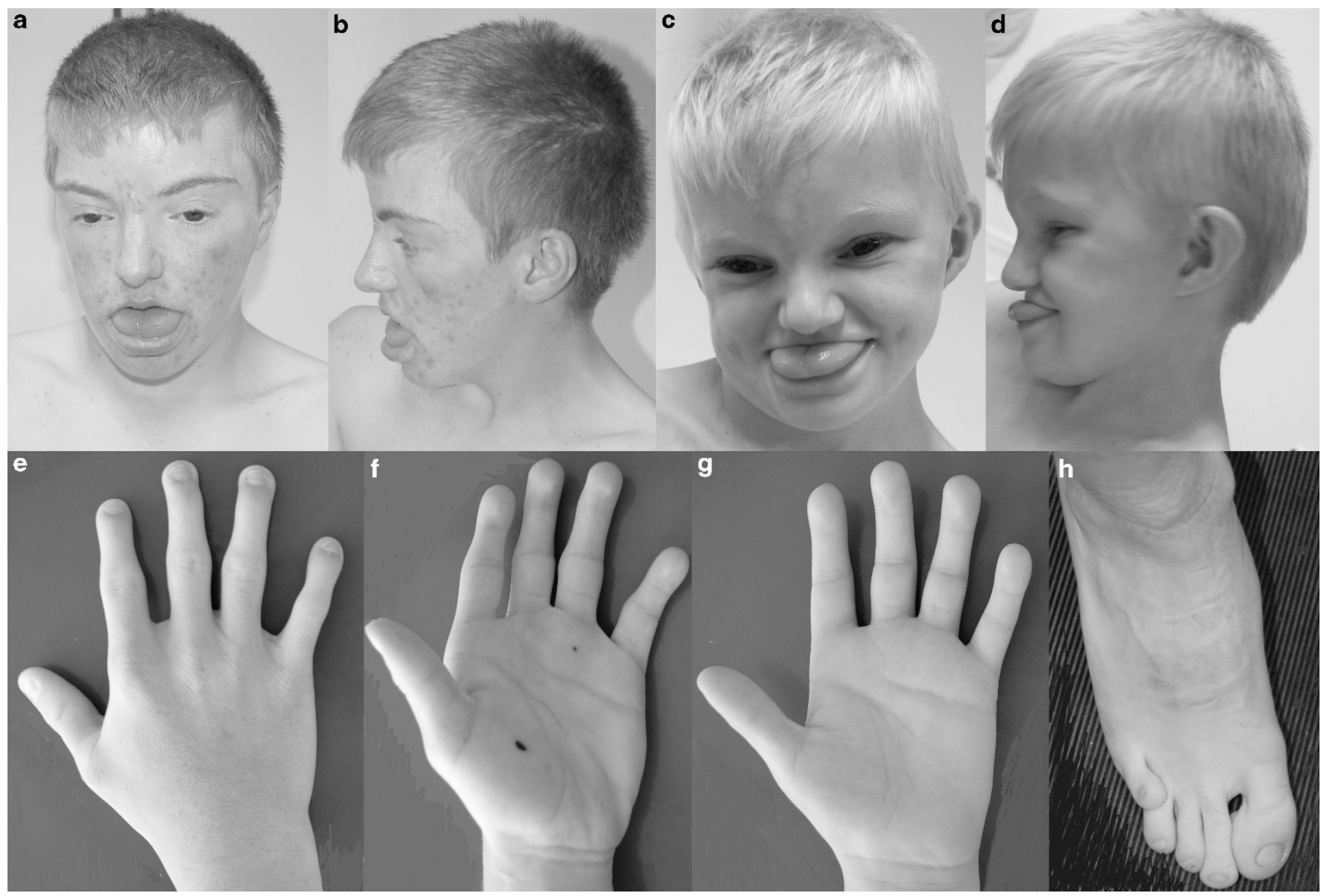

Figure 1 Facial photographs of patient 1 (a, b) and patient 2 (c, d) showing upslanting palpebral fissures, wide-set eyes, short philtrum, protruding tongue, and a broad neck. Hand anomalies of patient $1(\mathbf{e}, \mathbf{f})$ and patient $2(\mathbf{g})$. Note camptodactyly, reduced distal creases of the second to fifth fingers, clinodactyly of the fifth fingers, and hypoplastic finger nails of the second to fifth fingers. Foot of patient 1 (h) showing a broad hallux and an overriding fifth toe.

communicate in simple, dysgrammatic phrases with poor articulation, while receptive speech is well preserved. Their common facial signs included a broad forehead, upslanting palpebral fissures, wideset eyes, a short philtrum, and a broad neck with a low posterior hair line (Figures $1 \mathrm{a}-\mathrm{d}$ ). In addition, they had a large, protruding tongue. Their fingers showed camptodactyly and reduced distal creases of the second to fifth fingers, clinodactyly of the fifth fingers, fetal finger pads as well as hypoplastic finger nails of the second to fifth fingers (Figures 1e-g). Their halluces were broad and their toes were long with overriding fifth toes (Figure $1 \mathrm{~h}$ ).

We first performed 1.4 M aCGH in proband 1 to test for copy number variations that could potentially account for the observed phenotype. All results of this analysis were normal; therefore we decided to proceed with WES of DNA samples from both probands and their parents. Following sequence alignments, each data set contained $>130000$ variant calls and allowed for high-quality calls for over $90 \%$ of the human consensus coding sequence. We filtered variant call format files of the four family members by applying homozygous, compound heterozygous, and de novo filters in GeneTalk $^{8}$ and viewed variants using IGV browser. ${ }^{6}$ The only biologically relevant variant detected by homozygous filtering was a missense variant located on chromosome chrX.hg19:g.49106004 in exon 15 of the gene CCDC22 (NM_014008.4) at position c.1670A $>$ G; p.(Tyr557Cys) (Figure 1b). There were no variants annotated at this position in the exome variant server ((URL: http://evs.gs.washington. edu/EVS/) March 2014). The detected variant introduced an aminoacid exchange at the evolutionarily conserved position within a coiled-coil domain of the protein (Figures $2 \mathrm{~b}$ and $\mathrm{c}$ ). We performed segregation analysis and showed that the healthy brother and three other unaffected family members (father, maternal brother and maternal sister) did not harbor this variant, whereas the unaffected mother was a heterozygous carrier (Figure 2a).

To further explore the effect of the CCDC22 p.(Tyr557Cys) variant, we measured the expression of CCDC22 and WASH complex components in the LCLs derived from proband 1, his mother, and a control non-related healthy individual. Western blot analysis revealed a $50 \%$ decreased expression of CCDC22 in the patient cells as compared with those of the healthy mother and an unrelated control (Figure 3). Interestingly, we also detected an increased level of WASH1 expression in those cells, while the expression levels of strumpellin and FAM21 were not changed. Thus, p.(Tyr557Cys) variant negatively impacts CCDC22 protein stability and is associated with increased abundance of WASH1 protein. Transient expression of wild-type CCDC22 in HeLa cells resulted in a punctate distribution corresponding to vesicles (Figure 4). No clear overlap with the early endosome marker EEA1 or endocytosed EGF was observed. Expression of CCDC22-T17A, and CCDC22-Y557C revealed no significant differences in the subcellular distribution. 


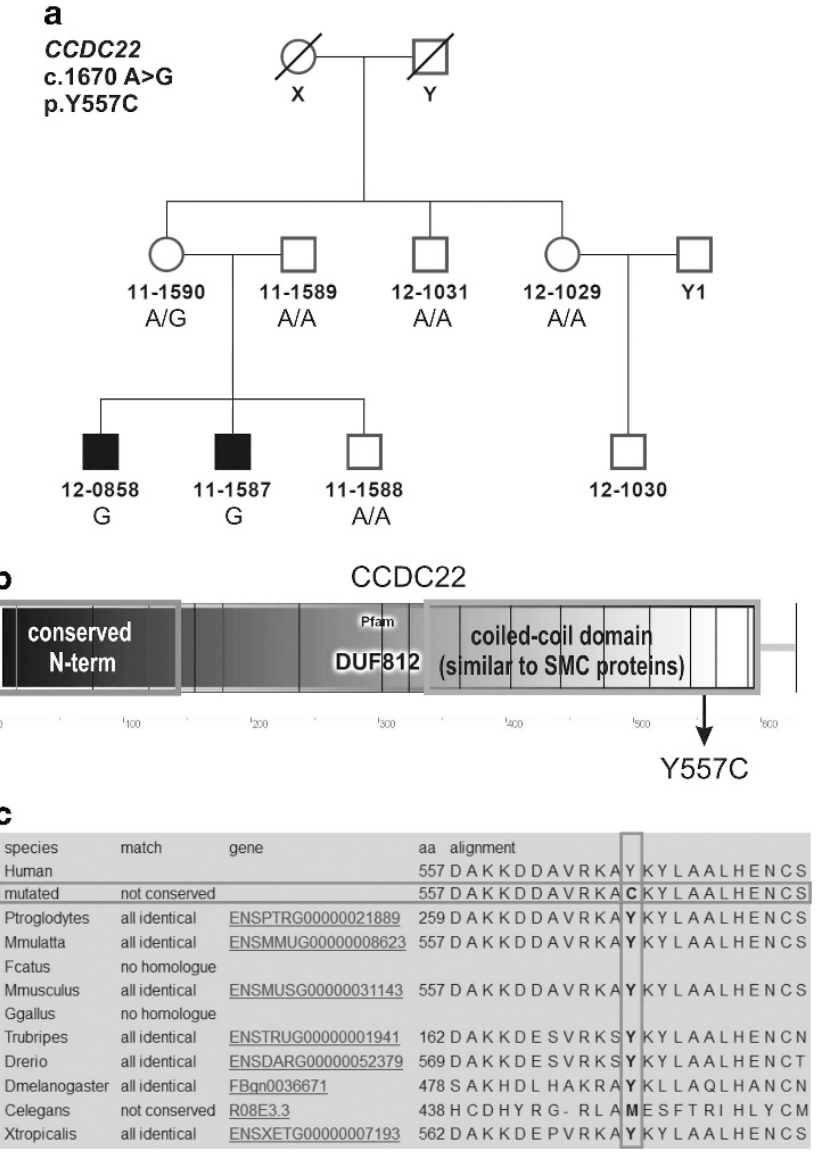

Figure $2 \mathrm{~A}$ pedigree of the family affected by the CCDC22 variant (a) Occurrence of the c.1670A $>$ G variant in the family affected with RSS/ $3 \mathrm{C}$ syndrome. The CCDC22 genotypes are annotated below each tested family member. (b) Schematic representation of the CCDC22 protein structure with annotated functional domains and the location of the p.Y557C variant within the coiled-coil domain of the protein. (c) Evolutionary conservation of the mutated amino-acid residue in the CCDC22.

\section{DISCUSSION}

The presented study documents a variant in CCDC22 gene being a cause of syndromic form of ID classified by us as similar to RSS/3C syndrome. A different CCDC22 variant has been recently shown to be a cause of X-linked ID (XLID) and involvement of CCDC22 in ID etiology awaited independent confirmation., ${ }^{9,10}$

CCDC22 is a highly conserved and broadly expressed protein that has been shown to interact through its $\mathrm{N}$-terminal part with COMMD proteins. ${ }^{11}$ COMMD proteins take part in multiple processes including regulation of NF-kB signaling, copper export from the liver, HIV infection, and sodium transport. ${ }^{12}$ The CCDC22 deficiency or a hypomorphic variant in this gene that impairs the interaction with COMMD proteins has been shown to lead to blunted activation of NF-kB. ${ }^{11}$ CCDC22 was also shown to interact with copines, ${ }^{13}$ a family of calcium-dependent membrane-binding proteins, with Nance-Horan syndrome protein (NHS), ${ }^{14}$ which is an important regulator of actin cytoskeleton dynamics, ${ }^{15}$ as well as with FAM21, ${ }^{16}$ a crucial component of the WASH complex that is composed of four additional core proteins: SWIP, strumpellin, CCDC53, and WASH1. The WASH complex controls the polymerization of actin at the surface of endosomes through the activation of the Arp $2 / 3$ complex, a major actin nucleator. ${ }^{17}$ WASHdependent actin polymerization promotes scission of transport intermediates of the different endosomal routes and affects recycling, ${ }^{17}$ degradation, ${ }^{18}$ and retrograde pathways. ${ }^{19,20}$ Inactivating variants in the gene encoding the WASH subunit strumpellin has been recently shown to cause a form of RSS/3C syndrome. ${ }^{2}$ Variant in another component of the WASH complex, SWIP, was shown to be causal to non-syndromic ID. ${ }^{21}$ Thus, variants in WASH complex components can result in various forms of ID.

The splice site variant NM_014008.4; c.219A > G/p.(Thr17Ala) in the CCDC22 gene was previously shown to be a cause of syndromic XLID (OMIM 300859) in a large family with six affected males. ${ }^{9}$ This study did not specifically describe the phenotype of the affected individuals as RSS/3C syndrome; however, it provided clinical data according to which the phenotype overlaps with RSS/3C syndrome and includes: presence of ID, Dandy-Walker malformation, cerebellar
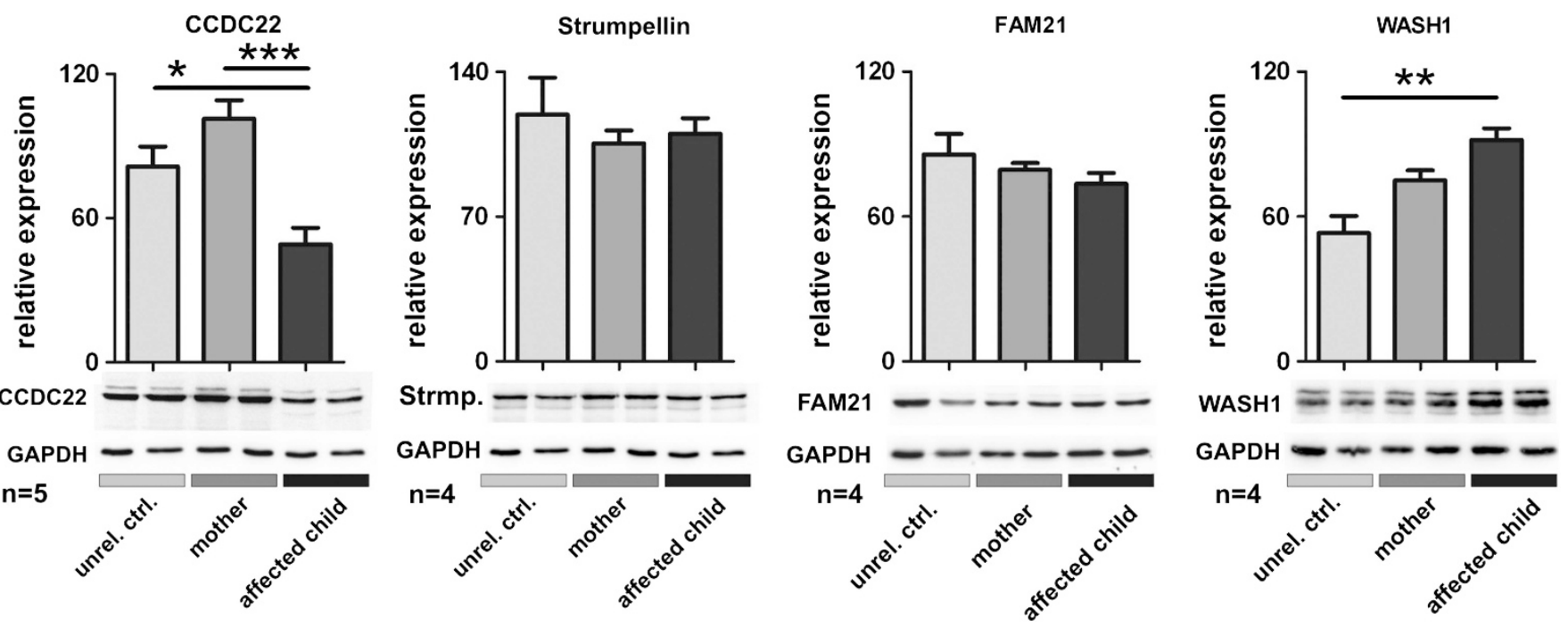

Figure 3 Western blot-based quantification of the CCDC22, strumpellin, Fam21, and Wash1 expression in the lymphoblastoid cell lines cells from affected patient, healthy mother, and non-related healthy control. Note a decreased expression of CCDC22 and increased expression of WASH1 in the patient cells. Statistical significance $-t$-test, ${ }^{*} P \leq 0.05 ;{ }^{* *} P \leq 0.01 ;{ }^{* *} P \leq 0.001$ 


\section{Flag-CCDC22 EEA1 EGF}

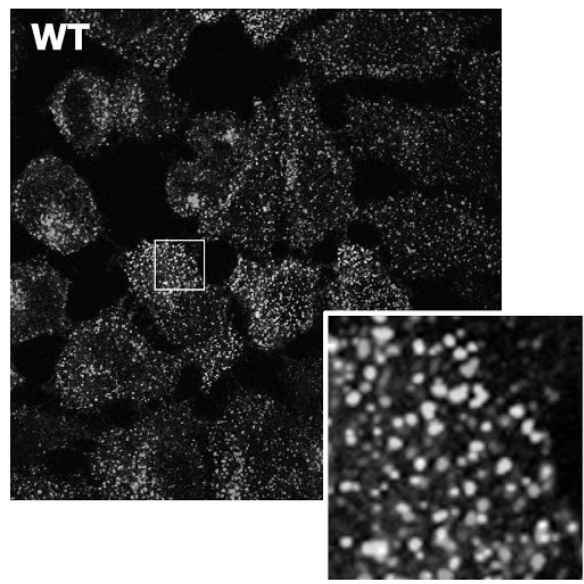

Flag-CCDC22 EEA1 EGF

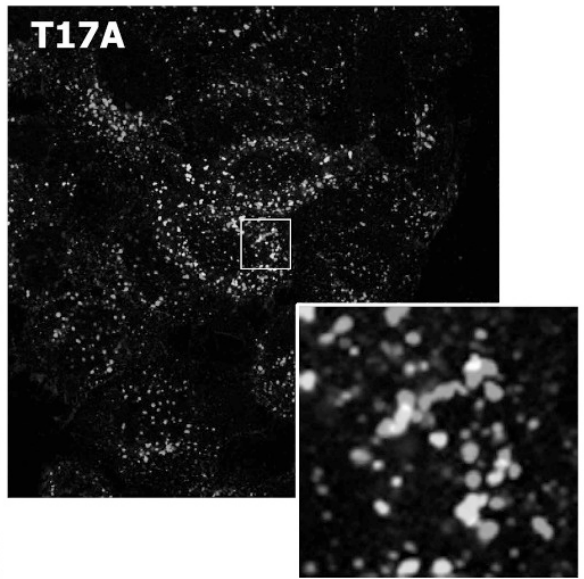

\section{Flag-CCDC22 EEA1 EGF}

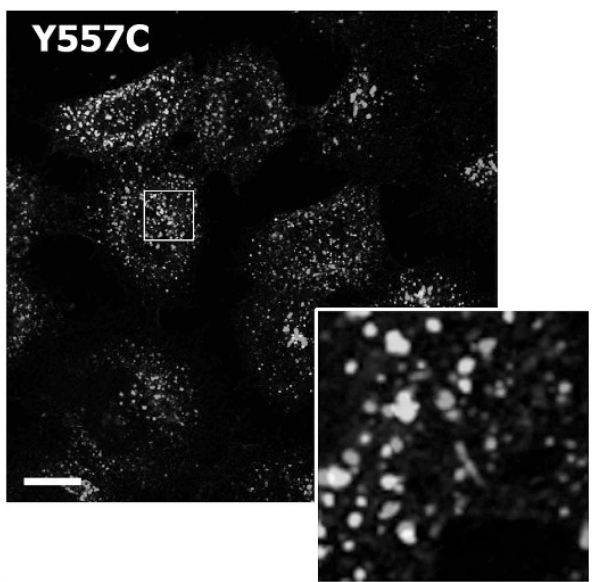

Figure 4 Subcellular distribution of CCDC22 variants associated with RSS/3C spectrum disorders upon EGF uptake. HeLa cells were transfected either with WT, T17A, or Y557C Flag-tagged CCDC22 vectors and subjected to EGF uptake. Cells were immunostained for EEA1 (in red), Flag (in green), and EGF fluorescence is presented in blue. Scale bar $=20 \mu \mathrm{m}$. The full colour version of this figure is available at European Journal of Human Genetics online.

hypoplasia, cardiac defects (atrial septum defect, patent ductus arteriosus, VSD, hypoplastic right pulmonary artery), specific facial features (hypertelorism, beaked nose and broad nasal tip, ear abnormalities, high arched palate), as well as various limb anomalies (see Table 1). In addition, two individuals were reported to show signs of ectodermal dysplasia, namely aplasia cutis and dental abnormalities. ${ }^{11}$ A series of other variants in CCDC22 was recently described in patients with non-syndromic XLID. ${ }^{11}$ These included the following missense variants: p.(Thr30Ala), p.(Arg128Gln), p.(Glu238Lys), p.(Arg321Trp), all localized in the N-terminal and mid part of the protein. Unfortunately no clinical data of the individuals affected with these variants were provided. Whereas the previously mentioned variant $\mathrm{p}$.(Thr17Ala) affected the interaction with COMMD1, none of the other variants disturbed this binding when tested in LCLs or after expression in HEK293 cells. ${ }^{11}$ Nevertheless, this study concluded that CCDC22 is required for the ubiquitination and subsequent turnover of $\mathrm{I} \kappa \mathrm{B}$ proteins, which is dependent on its interactions with COMMD proteins. As an additional evidence for altered NF-kB signaling, the signs of ectodermal dysplasia observed in a minority of patients were presented. Ectodermal dysplasia is known to result from of $\mathrm{I} \kappa \mathrm{B}-\alpha$ variants that alter its degradation or variants in the ectodysplasin pathway, a TNF-related signaling cascade that is involved in ectodermal development. ${ }^{11}$

The two patients described here show a broad phenotypic overlap with $\mathrm{RSS} / 3 \mathrm{C}$ syndrome described in the literature. In addition to ID, both displayed Dandy-Walker malformation, VSD, hand and foot anomalies (camptodactyly, clinodactyly, reduced distal creases of fingers, hypoplastic finger nails, and overriding toes), and facial dysmorphism. These two probands show a hemizygous variant in the CCDC22 gene that is localized to the C-terminally located coiled-coil domain. Although this variant can potentially have different effects on the protein function and partner protein interactions than the $\mathrm{N}$-terminally localized p.(Thr17Ala) variant, the observed phenotypic features in both families are strikingly similar and consistent with the diagnosis of RSS/3C syndrome (Table 1). However, it should be noted that the short stature and relative large head circumference are not mentioned for the individuals carrying the CCDC22 variant p.(Thr17Ala).

In the light of the recent report about strumpellin intronic variants $(\mathrm{c} .3335+2 \mathrm{~T}>\mathrm{A}, \mathrm{c} .3335+4 \mathrm{C}>\mathrm{A}$ and $\mathrm{c} .3335+8 \mathrm{~A}>\mathrm{G})$ being causal to a form of RSS/3C syndrome, ${ }^{2}$ it is interesting to note that CCDC22 was shown to interact with FAM21, a subunit of the WASH complex. ${ }^{16}$ Apart from ID, phenotypic overlap in both patient groups consists of the same pattern of cerebellar and cardiac as well as hand and foot anomalies (Table 1). However, facial dysmorphisms in both groups are different. Whereas individuals with homozygous KIAA0196 sequence variants show macrocephaly, down slanting palpebral fissures, and low set ears, the patients reported here present with different facial features, for example, occipitofrontal circumference in the upper normal range, upslanting palpebral fissures, and normal appearing ears (Figures 1a-d). Furthermore, the affected individuals of the family reported here display postnatal short stature in contrast to normal growth in the affected families carrying the homozygous sequence variants of the KIAA0196 gene. Nevertheless, it seems likely that variants in CCDC22 affect the same molecular machinery as variants in strumpellin. Our observation that WASH1 expression level is increased in the CCDC22-mutant LCL cells supports the notion of the possible link between CCDC22 and WASH complex function.

One remaining question is how two missense variants (p.(Thr17Ala) and p.(Tyr557Cys)) in two very distant regions of the CCDC22 protein lead to such closely related phenotypes. As indicated by our immunoblot analysis, CCDC22-Y557C shows significantly reduced protein stability in LCLs from patients, which is likely caused by a premature or accelerated degradation of mutant protein, provoked by aberrations of its conformation. ${ }^{22}$ Likewise, the c.219 $A>G$ change leading to the very mild exchange $p$.(Thr17Ala) entails a splice defect, which is not unlikely to also lead to reduced levels of the wild-type protein. Unfortunately, no protein expression was investigated in this previous study. Another finding supporting this hypothesis is the comparable vesicular pattern of wild-type and mutated CCDC22 after transient expression, which, however, does not preclude functional changes independent of intracellular sorting events.

In conclusion, we show that $C C D C 22$ variants, leading to at least partial loss of the protein, are associated with ID, cerebellar anomalies, cardiac defects, facial dysmorphism, as well as hand and foot anomalies. Our findings widen the phenotypic as well as the genotypic spectra of syndromic XLID caused by variants in CCDC22. Together with previous reports this suggests that the clinical spectrum 
associated with $C C D C 22$ variants can range from syndromic manifestations similar to RSS/3C syndrome to non-syndromic ID. Given the marked clinical variability of RSS/3C syndrome and the heterogeneous etiology, we suggest to collectively call this group of conditions WASHopathies.

\section{CONFLICT OF INTEREST}

The authors declare no conflict of interest.

\section{ACKNOWLEDGEMENTS}

We acknowledge the members of the family for their participation in this study. We thank Monika Osswald, Catrin Janetzki, and Gundula Leschik for technical assistance with DNA preparation, exome enrichment, and highthroughput sequencing and Phillipe Schröter for Sanger sequencing.

\section{AUTHOR CONTRIBUTIONS}

MK: analyzed data, wrote the manuscript; PK: analyzed data, provided bioinformatics support; JH: coordinated NGS; AH, CS: performed the experiments; MM: provided molecular expertise, reviewed the manuscript; KM: conducted western blots; DE: reviewed the manuscript; KK: reviewed the manuscript; GG and SU: studied the patients, collected the samples, helped in the clinical work; BP, GG, and SU: contacted the family, collected clinical material, and reviewed the manuscript; UK: reviewed the manuscript; PNR: provided bioinformatics support, SM: supervised the experimental work, provided financial support for the research and critically revised the manuscript; DH: analyzed the clinical data and wrote the manuscript.

\section{ETHICS APPROVAL}

Patient consent was obtained for this study. Ethics approval was granted by the Institutional Review Board.

1 Leonardi ML, Pai GS, Wilkes B, Lebel RR: Ritscher-Schinzel cranio-cerebello-cardiac (3C) syndrome: report of four new cases and review. Am J Med Genet 2001; 102: 237-242.

2 Elliott AM, Simard LR, Coghlan G et al: A novel variant in KIAA0196: identification of a gene involved in Ritscher-Schinzel/3C syndrome in a First Nations cohort. J Med Genet 2013; 50: 819-822.

3 Craft E, Wildig CE, Crow YJ: 3C syndrome. Am J Med Genet A 2010; 152A: 1026-1027.

$4 \mathrm{Li} \mathrm{H}$, Handsaker B, Wysoker A et al: The Sequence Alignment/Map format and SAMtools. Bioinformatics 2009; 25: 2078-2079.
5 Wang K, Li M, Hakonarson H: ANNOVAR: functional annotation of genetic variants from high-throughput sequencing data. Nucleic Acids Res 2010; 38: e164.

6 Thorvaldsdottir H, Robinson JT, Mesirov JP: Integrative genomics viewer (IGV): high-performance genomics data visualization and exploration. Brief Bioinform 2013; 14: 178-192.

7 Baasanjav S, Jamsheer A, Kolanczyk M et al: Osteopoikilosis and multiple exostoses caused by novel variants in LEMD3 and EXT1 genes respectively-coincidence within one family. BMC Med Genet 2010; 11: 110 .

8 Kamphans T, Krawitz PM: GeneTalk: an expert exchange platform for assessing rare sequence variants in personal genomes. Bioinformatics 2012; 28: 2515-2516.

9 Voineagu I, Huang L, Winden $\mathrm{K}$ et al: CCDC22: a novel candidate gene for syndromic X-linked intellectual disability. Mol Psychiatry 2012; 17: 4-7.

10 Piton A, Redin C, Mandel JL: XLID-causing variants and associated genes challenged in light of data from large-scale human exome sequencing. Am J Hum Genet 2013; 93: 368-383.

11 Starokadomskyy P, Gluck N, Li $\mathrm{H}$ et al: CCDC22 deficiency in humans blunts activation of proinflammatory NF-kappaB signaling. J Clin Invest 2013; 123: 2244-2256.

12 Burkhead JL, Morgan CT, Shinde U, Haddock G, Lutsenko S: COMMD1 forms oligomeric complexes targeted to the endocytic membranes via specific interactions with phosphatidylinositol 4,5-bisphosphate. J Biol Chem 2009; 284: 696-707.

13 Tomsig JL, Snyder SL, Creutz CE: Identification of targets for calcium signaling through the copine family of proteins. Characterization of a coiled-coil copine-binding motif. J Biol Chem 2003; 278: 10048-10054.

14 Coccia M: Molecular genetics of Nance-Horan syndrome and X-linked cataract. Doctoral thesis, Department of Molecular and Cellular Neuroscience, Institute of Ophthalmology, University College, London, 2010.

15 Brooks SP, Coccia M, Tang HR et al: The Nance-Horan syndrome protein encodes a functional WAVE homology domain (WHD) and is important for co-ordinating actin remodelling and maintaining cell morphology. Hum Mol Genet 2010; 19: 2421-2432.

16 Harbour ME, Breusegem SY, Seaman MN: Recruitment of the endosomal WASH complex is mediated by the extended 'tail' of Fam21 binding to the retromer protein Vps35. Biochem J 2012; 442: 209-220.

17 Derivery E, Sousa C, Gautier JJ, Lombard B, Loew D, Gautreau A: The Arp2/3 activator WASH controls the fission of endosomes through a large multiprotein complex. Dev Cell 2009; 17: 712-723.

18 Duleh SN, Welch MD: WASH and the Arp2/3 complex regulate endosome shape and trafficking. Cytoskeleton (Hoboken) 2010; 67: 193-206.

19 Gomez TS, Billadeau DD: A FAM21-containing WASH complex regulates retromerdependent sorting. Dev Cell 2009; 17: 699-711.

20 Harbour ME, Breusegem SY, Antrobus R, Freeman C, Reid E, Seaman MN The cargo-selective retromer complex is a recruiting hub for protein complexes that regulate endosomal tubule dynamics. J Cell Sci 2010; 123: 3703-3717.

21 Ropers F, Derivery E, Hu H et al: Identification of a novel candidate gene for non-syndromic autosomal recessive intellectual disability: the WASH complex member SWIP. Hum Mol Genet 2011; 20: 2585-2590.

22 Waters PJ: Degradation of mutant proteins, underlying 'Ioss of function' phenotypes, plays a major role in genetic disease. Curr Issues Mol Biol 2001; 3: 57-65. 